\title{
Estudio del perfil de ciclo básico de la carrera de Trabajo Social de la Universidad Católica Silva Henríquez
}

\author{
María Luisa Díaz Letelier
}

\section{RESUMEN}

El documento presenta algunos resultados de un estudio realizado el 2010 , en torno a la evaluación del Perfil Intermedio I del Plan de Formación de la carrera de Trabajo Social de la Universidad Católica Silva Henríquez, orientado por competencias. Este estudio corresponde a un proyecto piloto de evaluación de una propuesta de innovación curricular en la educación superior en un contexto complejo de cambio de época.

Palabras clave: Formación por competencias - plan de formación evaluación

\section{Estudo do perfil de ciclo básico da carreira de Trabalho Social da Universidade Católica Silva Henríquez}

\author{
RESUMO
}

O artigo apresenta alguns resultados de um estudo realizado em 2010 em relação à avaliação do Plano de intermediários perfis de formação I da carreira de Trabalho Social na Universidad Católica Silva Henríquez, guiado por competências. Este estudo é uma avaliação-piloto de uma inovação curricular proposta no ensino superior em um contexto complexo da mudança dos tempos

Palavras chave: Baseada em competências de formação - Plano de formação - avaliação

Study of the profile of basic cycle of the career of Social Work of the Catholic University Silva Henríquez

\section{ABSTRACT}

The paper presents some results of a study conducted in 2010, regarding the evaluation of Intermediate I Profile of the Training Plan of the career of Social Work at the Universidad Católica Silva Henríquez, skills-oriented. This study is a pilot project for evaluating a proposal for curriculum innovation in higher education in a complex context of changing times.

Key words: Competencybased training - training plans - evaluation.

* Chilena. Trabajadora Social. Docente de la Escuela de Trabajo Social. Universidad Católica Silva Henríquez.Correo electrónico: mldiaz@ucsh.cl. 


\section{Antecedentes}

El año 2007 la carrera de Trabajo Social de la Universidad Católica Silva Henríquez (UCSH) dio inicio a un nuevo Plan de Formación orientado por competencias, atendiendo a las directrices y legado valórico ofrecido por el Modelo Formativo y la misión de esta institución (UCSH, 2003).

Este Plan, se asume como una innovación curricular que busca dar respuesta a los requerimientos de una sociedad globalizada y compleja, y, más particularmente, aportar al enfrentamiento de los desafíos de la educación superior en este contexto: "reconoce la necesidad de un trabajo social que: desarrolla un especial esfuerzo en torno a la generación de una recomprensión de lo social; devela los conflictos sociales subyacentes a los problemas sociales; propicia y gestiona estrategias participativas atingentes a los sectores más excluidos y vulnerados de la sociedad; innova metodológicamente de modo de abordar los nuevos problemas sociales adheridos a las transformaciones globales; despliega acciones tendientes a la búsqueda de nuevas relaciones de convivencia social, favorecedoras de un entorno democrático; estimula y genera espacios de conversación profesional y disciplinar; sustenta su quehacer desde un compromiso ético." (Escuela de Trabajo Social, UCSH, 2006:6).

Desde ese modelo de formación y enfoque curricular, se concibe la evaluación como una acción a disposición de los aprendizajes, y constituyente de una óptica transformadora en el quehacer pedagógico. Por tanto, se asume como un componente más del modelo de formación, que debiera instalarse como parte inmanente y permanente del proceso de formación profesional, reconociéndolo como facilitador de retroalimentación y definición de estrategias de mejoramiento. En este sentido, se le visualiza como un elemento necesario en la instalación de mecanismos de aseguramiento de la calidad de la educación en la formación universitaria.

El Plan de Formación de Trabajo Social de la UCSH supone un Perfil de Egreso, así como dos perfiles intermedios: Perfil Intermedio I, al término del primer ciclo de formación (vale decir, luego de la consecución del cuarto semestre de la carrera); y, Perfil Intermedio II, al término de la Licenciatura en Trabajo Social (al término del sexto semestre).

En ese escenario, en el año 2009 se da inicio a este proceso evaluativo, diseñando un Estudio en torno a la evaluación del Perfil Intermedio I, como Proyecto piloto, financiado y asesorado por la Dirección de Docencia (DIDOC) de la UCSH. 
El objetivo general fue "Determinar el estado de desarrollo de algunas de las competencias comprendidas en el perfil intermedio del ciclo básico de la carrera de Trabajo Social de la UCSH, en los estudiantes de las generaciones 2007 y 2008, jornadas diurna y vespertina".

\section{Marco referencial}

El estudio atiende a un contexto social que a juicio de varios autores (Aquín, 2004; Garretón, 2000; Lechner, 2002) reconoce grandes transformaciones a nivel mundial, de las cuales emergen nuevos desafíos y requerimientos para el quehacer del trabajo social. Particularmente, se hace referencia a: las transformaciones económicas $^{1}$ (globalización de las economías nacionales, flexibilización y desregulación de los mercados), transformaciones sociales (cambios demográficos que modifican la estructura de la población de los países, movimientos migratorios cada vez más fluidos, exclusión social, configuración de una nueva cuestión social y surgimiento de la neofilantropía), transformaciones culturales (proceso acelerado de individualización, cultura del consumo que impacta en los estilos de convivencia, heterogeneización y segunda hibridización de la cultura, mediatización de la comunicación social, cambios en la configuración de las familias, transformación subterránea del ser hombre y mujer, convivencia social deteriorada); transformaciones tecnológicas (revolución biotecnológica, revolución de la informática y la comunicación).

Así, se visualiza que el escenario de transformaciones económicas, sociales, culturales y tecnológicas antes aludido tiene un correlato en la configuración de un marco educativo también de transformaciones. El surgimiento de la sociedad de la información trae aparejado el nacimiento de las sociedades de aprendizaje (González y López, 2004; UNESCO, 2005).

En este contexto, la educación superior asiste a una serie de transformaciones que se han instalado en la sociedad globalizada y compleja en la cual nos desenvolvemos. Esto implica para las universidades algunos desafíos a enfrentar. A saber, en palabras de Zabalza (2002), adaptarse a las demandas del empleo; ubicarse en un contexto de competitividad que requiere actuar con calidad y capacidad de cambio; mejorar la gestión ante la disminución de recursos públicos; incorporar nuevas tecnologías, en la gestión y

1 Subrayado de la autora. 
docencia; convertirse en impulsora del desarrollo local; potenciar la interdisciplinariedad, dominio de lenguas extranjeras, movilidad de docentes y estudiantes, otros.

En particular en las universidades latinoamericanas, las transformaciones más significativas en los últimos años dicen relación con: "i) la gran expansión cuantitativa del sector; ii) su notable diversificación institucional; iii) el aumento de la participación del sector privado; iv) el incremento de la internacionalización; v) el cambio de actitud de los gobiernos; vi) los esfuerzos de transformación de algunas universidades". (Audibert, Carrasco, Desimone, 2009: 59).

Ante estas transformaciones y desafíos, la educación superior busca dar respuesta a través de diversas estrategias, siendo una de ellas el campo de la innovación curricular. Una de las propuestas planteadas en éste refiere a formación profesional bajo el modelo de competencias, como "orientación educativa que pretende dar respuestas a la sociedad del conocimiento (...)" (Zúñiga; Poblete y Vega: 2009).

Acerca de las competencias se han planteado diversas concepciones y perspectivas. La discusión al respecto pareciera que recién se instala en la educación superior. Tobón reconoce cuatros enfoques para la conceptualización de éstas (Tobón, 2008):

- Enfoque conductista: se les reconoce como comportamientos clave de las personas para alcanzar competitividad en las organizaciones.

- Enfoque funcionalista: las traduce como atributos que requieren las personas para confluir con los propósitos de procesos laborales/profesionales pre-definidos.

- Enfoque constructivista: las visualiza como habilidades, conocimientos, y destrezas, para resolver -desde una perspectiva organizacional-dificultades en procesos laborales/ profesionales.

- Enfoque complejo: procesos complejos en los que se evidencia un desempeño en actividades y en la resolución de problemas en forma idónea y desde una perspectiva ética, en pro de realización personal, calidad de vida, en un marco de desarrollo sostenible y sustentable.

A su vez, en la formación por competencias se observan también diversos modelos (Zúñiga, Poblete y Vega: 2009): 
- Modelos que diferencian e integran las competencias genéricas con las específicas disciplinares (Ejemplo de ello son: Tuning, Bélgica, Alemania, Holanda).

- Modelos mixtos, que entremezclan las competencias genéricas y áreas disciplinares (España, Portugal, Francia, otros).

- Modelos que no diferencian las competencias (Italia, Finlandia).

En Latinoamérica se ha asumido también la formación por competencias. Un impulso a este esfuerzo fue dado por el proyecto Tuning latinoamericano, ya finalizado. Sin embargo, no es posible identificar una cierta homogeneidad en el enfrentamiento de este desafío ni menos un trabajo conjunto, a diferencia de lo ocurrido en Europa (Audibert, M.; Carrasco, R.; Desimone, P. 2009).

La formación por competencias supone entre otras cuestiones, plantearse el tema de una evaluación permanente, que se instale como mecanismo de retroalimentación y generador de insumos para la verificación de los resultados y calidad alcanzada.

Poblete y Villa (2007) reconocen cuatro elementos centrales en la formación por competencias: 1) estrategias y metodologías de enseñanza-aprendizaje pertinente; 2) Modalidad o forma de organizar el proceso (presencial, semi-presencial, virtual); 3) seguimiento del aprendizaje, 4) sistema variado de evaluación: saber qué se va a evaluar, definir cómo se evaluará; concretar el nivel de logro que se evaluará.

De hecho, implica un replanteamiento respecto del sentido de la evaluación, sus modalidades y usos. De modo de transitar desde una evaluación centrada en la calificación del estudiante a una evaluación que implique un seguimiento sostenido de, al menos: procesos de aprendizaje del conjunto de los estudiantes en formación, trayectoria de cada uno de ellos, coherencia y consistencia de la propuesta de formación, recursos de aprendizaje usados por los estudiantes, relación y proceso producido en actividades y dispositivos pedagógicos clave.

Es así que podemos reconocer tres ámbitos de evaluación en la formación por competencias (Alvarado, Cárcamo y García 2009: 111):

- Ámbito de planeación estructural: donde el centro de la evaluación está colocado en la efectividad del perfil de egreso y de la estructura global del currículum y plan formativo; 
- Ámbito de planeación específico: cuyo énfasis está ubicado en los logros de aprendizaje de cada módulo o actividad curricular.

- Ámbito de planeación transversal: en el cual el centro refiere a observar y dar cuenta de ciertos momentos de la formación definidos con antelación, con el propósito de: producir información respecto de los aprendizajes comprometidos en estos niveles (dejando atrás la lógica de aprendizajes por actividad curricular), analizar esta información para tomar decisiones que permitan sopesar los avances y estado en que se encuentran los estudiantes respecto del perfil de egreso planteado; formular medidas de mejora.

En este estudio se asume una evaluación en el ámbito de planeación transversal, tendiente a producir información relativa a niveles de logro alcanzados por los estudiantes en función de un momento determinado de su formación profesional: primer ciclo de formación o ciclo básico. Es decir, evaluación centrada en detectar el estado de los ejes de progresión en la trayectoria de aprendizaje pre-definida.

\section{Metodología}

\section{Población:}

La evaluación de Perfil Intermedio I de la carrera de Trabajo Social de la UCSH consideró a los/as estudiantes de las generaciones 2007 y 2008, de las jornadas diurna y vespertina, que contaban con la calidad de alumnos regulares de la carrera, y que habían aprobado las actividades curriculares del Plan de Especialidad consideradas entre el primer y el cuarto semestre. Rindieron la evaluación 57 estudiantes: $65,51 \%$ del total.

\section{Fases de Trabajo}

\subsection{Identificación de Competencias Relevantes:}

Se optó por realizar una selección de las competencias estimadas como más relevantes para el logro de este Perfil, así como las más viables de evaluar en este proceso.

Con este propósito, se llevó a cabo una consulta a los docentes de planta y a los docentes adjuntos de la carrera. Las competencias más relevadas por los docentes fueron: 
Competencia 1: "Ejercita una actitud indagativa y reflexiva, interesándose por actividades de estudio en su campo profesional, lo cual concreta a través del desempeño activo en trabajos intermedios de investigación social."

Competencia 2: "Analiza procesos de intervención social y ejercita el diseño de estrategias pertinentes a realidades específicas que promuevan la responsabilidad social, aplicando contenidos conceptuales y metodológicos del campo de la intervención social, desde una perspectiva ética y en observancia a los valores de la UCSH, con énfasis en el respeto a los derechos humanos, la justicia social, igualdad, democracia, respeto de las personas."

Competencia 3: "Se reconoce como sujeto activola de sus procesos de aprendizaje, participando en procesos de aprendizaje autónomo y cooperativo, y siendo un/a estudiante críticola y auto-reflexivola, que incorpora la auto evaluación como una forma de enriquecimiento de su proceso de formación profesional."

Sin embargo, para efectos de este proceso se desestimó la evaluación de la Competencia 3, en tanto requiere un tratamiento diferente desde instrumentos de autopercepción y procesos de observación sistemática. De allí que se eligen sólo la Competencia 1 y Competencia 2. Posteriormente, se identificaron las actividades curriculares del Plan de Especialidad asociadas a dichas competencias, por eje formativo.

\subsection{Definición de Especificaciones:}

Al ser una evaluación de carácter cognitivo, se incluyeron tres formas de pensamiento:Interpretativo (Ubicación y comprensión de aspectos y relaciones que estructuran contextos y problemáticas específicas); Argumentativo (Exposición de razones fundamentadas en un saber profesional que permitan contrastar, demostrar, criticar, explicar, satisfactoriamente situaciones o escenarios de intervención e investigación social); Propositivo (Elaboración de alternativas de explicación y/o de acción ante situaciones y fenómenos, particularmente frente a situaciones a enfrentar en el ámbito del ejercicio profesional. A su vez, se incorporaron dos dimensiones de estas competencias o campos del saber, estimándolas factibles de evaluar con este tipo de instrumento: saber conocer y saber hacer.

Finalmente, se definió la distribución de ítems en una Tabla de Especificaciones, buscando un cierto equilibrio respecto de la representación y pesos asignados. 


\subsection{Diseño de ítems, Juicio de Expertos y Corrección de Ítemes:}

Se buscó que la elaboración de ítems lograra articular: las competencias relevadas como importantes, las dimensiones (o campos del saber), y las formas de pensamiento de estas competencias, con los aprendizajes esperados definidos por las actividades curriculares comprometidas en su logro. Los ítems elaborados, fueron sometidos a juicio de experto y, posteriormente corregidos conforme a las sugerencias de ese proceso.

\subsection{Elaboración de Prueba Tipo.}

Se elaboró un Instrumento único, con los 30 ítems seleccionados y corregidos, de opción múltiple y más de una respuesta. A él se adosó un Cuestionario de caracterización de la población participante del estudio.

\section{Procesamiento cuantitativo ${ }^{23}$}

\subsection{Modelo utilizado para el procesamiento de datos}

El modelo de procesamiento de datos usado es el Modelo de Rasch (con software Winsteps), situado desde la Teoría Respuesta al Ítem (TRI). Este modelo considera que la respuesta a un ítem sólo depende de la interacción entre la habilidad del sujeto y de la dificultad del ítem b. Desde la perspectiva de este modelo el valor obtenido de la habilidad de una persona es independiente de los ítems utilizados en una prueba en particular.

El análisis por el modelo de RASCH construye mediciones lineales de la habilidad de las personas y la dificultad de las preguntas, al mismo tiempo que establece índices de la precisión y exactitud de la medición (ajuste). Este modelo especifica que cada respuesta útil en una prueba surge de la interacción probabilística lineal entre la medida de la habilidad de una persona y la medida de la dificultad de una pregunta. Una forma simple de expresar este modelo es:

2 Este acápite ha sido elaborado por el Dr. Carlos Pardo Adames, Asesor Subdirección de Estadísticas, Instituto Colombiano para la Evaluación de la Educación - ICFES, Colombia.

3 El procesamiento cuantitativo de los datos se desarrolló con la Consultoría del Dr. Carlos Pardo Adames, especialista en aplicación de la Teoría de Respuesta al Ítem, Modelo de Rasch. 
$\log \frac{\text { probabilidad de éxito }}{\text { probabilidad de fracaso }}=\frac{\text { habilidad de la persona }}{\text { dificultad de la pregunta }}$

\subsection{Indicadores estadísticos y psicométricos para análisis}

Se realizó un análisis de ítem cuyo criterio principal fue el valor de ajuste, que indica la correspondencia entre un grupo de datos y el modelo estadístico utilizado para representarlos. El ajuste próximo (infit) refiere a la relación entre los datos que se encuentran cerca del valor de dificultad del ítem y el valor de dificultad; el ajuste lejano refiere a la relación de los datos que se encuentran lejos de dicho valor de dificultad y esa dificultad. Ambos indicadores se calculan para la clave.

Se consideró: Dificultad; Ajuste; Error Estándar de Medición; Análisis de Prueba; Media; Desviación Estándar; Confiabilidad.

\subsection{Características psicométricas del Instrumento}

Luego del procesamiento se generó una tabla con la información de cada ítem de acuerdo con los indicadores mencionados anteriormente. De acuerdo con los criterios establecidos, todos los ítems son de buena calidad técnica y no fue necesario replantearlos.

A su vez, se obtuvieron los diferentes indicadores estadísticos para la prueba, la que se encuentra bien ajustada a la población que aborda: es adecuada para los estudiantes ya que el valor de la media es muy cercano a cero $(0,28)$ y la diferencia es menor a una desviación estándar (0.65). La desviación estándar indica que la población es homogénea, al ser inferior a 1. Esta homogeneidad explica por qué la confiabilidad es algo baja $(0,55)$, pero dentro del límite de lo aceptable.

En conclusión, los ítems de la prueba y la prueba en sí misma es de muy buena calidad técnica psicométrica y cumple con la función para la que fue diseñada.

\subsection{Niveles de logro}

Luego de calibrar los ítems de la prueba utilizada se produjeron los resultados de cada estudiante. Estos son:

- Total puntaje escala: El resultado producido por el software en la escala logit del Modelo de Rasch se transformó a una escala estándar con promedio de 50 puntos y desviación estándar de 10 puntos. 
- Competencia 01 Escala Final; Competencia 02 Escala Final; Dimensión del Conocer Escala Final; Dimensión del Hacer Escala Final: El resultado producido por el software en la escala logit del Modelo de Rasch se transformó a escala estándar con promedio de 5 puntos y desviación estándar de 1 punto, utilizando los mismos parámetros de la TOTAL PUNTAJE ESCALA. En este sentido, los resultados de todas las escalas son comparables entre sí.

- Nivel Total: A partir de la escala producida por el modelo de Rasch, se seleccionaron los puntos de anclaje de los diferentes niveles para el resultado total en la prueba. La tabla de correspondencia de los puntos de anclaje para la definición de niveles es la siguiente:

\begin{tabular}{|c|c|c|}
\hline PUNTO ANCLAJE & PUNTAJE RASCH & PUNTAJE TOTAL \\
\hline I & -1 & 30 \\
\hline II & -0.2 & 45 \\
\hline III & 0.6 & 55 \\
\hline IV & 1.4 & 67 \\
\hline
\end{tabular}

Con base en estos puntos se obtuvo el nivel para cada estudiante. Para definir los niveles de: Competencia 1; Competencia 2; Dimensión del Conocer; Dimensión del Hacer; se utilizaron los mismos puntos de anclaje del resultado total.

Esquema Niveles de Logro

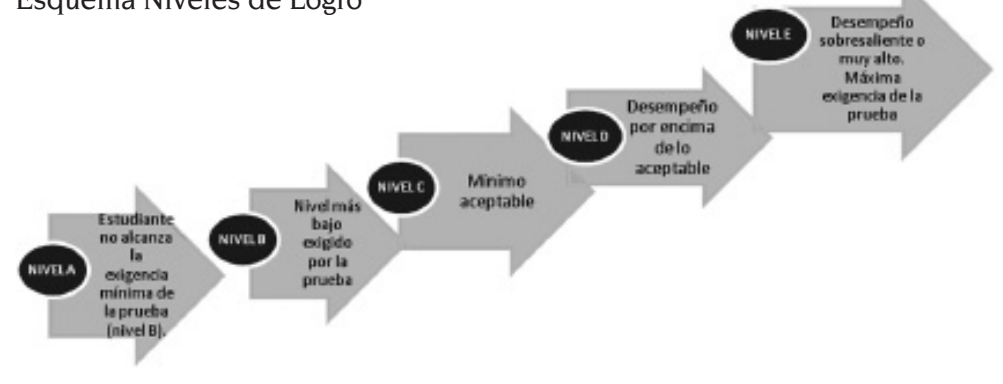

\section{Resultados}

El promedio total alcanzado por el grupo de estudiantes sometido a evaluación alcanza los 50 puntos. Este promedio se comporta de modo bastante similar por generaciones, observándose variaciones muy mínimas: logro de $+2,35$ puntos en la generación 2008 por sobre la generación 2007; traduciéndose en: 49,01 puntos en la generación 2007 y 51,36 en la generación 2008.

De acuerdo con la definición de niveles de logro, y según se muestra en el Gráfico 1, los estudiantes muestran una concentración en el 
nivel C con un 45,6\% (26 estudiantes). Al considerar la distribución en los niveles iguales al mínimo aceptable y superiores, vale decir nivel C, D y E, se constata que se concentra el 73,7\% (42 casos). En los niveles superiores, nivel D y E, se encuentra un 28,1\% ( 16 casos). En los niveles más bajos, nivel A y B, se ubica un 26,3\% (15 casos).

Al atender a los logros por niveles para cada una de las dos competencias evaluadas, se aprecia para la competencia 1 un comportamiento similar al total, según se observa en el Gráfico 1. Es así que un 73,7\% (42 casos) alcanza un logro igual o mayor que el mínimo aceptable (Niveles C, D, y E), apreciándose una concentración de un 43,9\% (25 casos) en el nivel C. En los dos niveles superiores, nivel D y E, un 29,8\% (17 casos). En los niveles más bajos (Nivel A y B) se encuentra un 26,4\% (15 casos).

\section{Resultado Competencia 1 por niveles de logro}

Gráfico 1

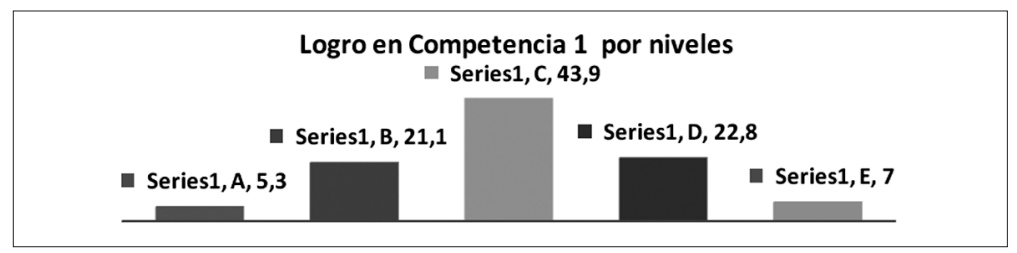

Como muestra la gráfica 2, cuando se trata de la competencia 2, se observa que el 70,2\% (40 casos) alcanza un desempeño igual o superior al mínimo aceptable (nivel C, D y E). Existe, respecto de la competencia 1, una menor concentración en el nivel C. Es así que en éste se ubica sólo un 35,1\% (20 casos); -8,8 que en la Competencia 1. Aumentando en los niveles superiores, nivel D y E, con un 35,1\% (20 casos). Así también se observa un aumento, aunque menor, del porcentaje de estudiantes en los niveles inferiores, nivel A y B, con un 29,8\% (17 casos).

\section{Resultado Competencia 2 por niveles de logro}

Gráfico 2

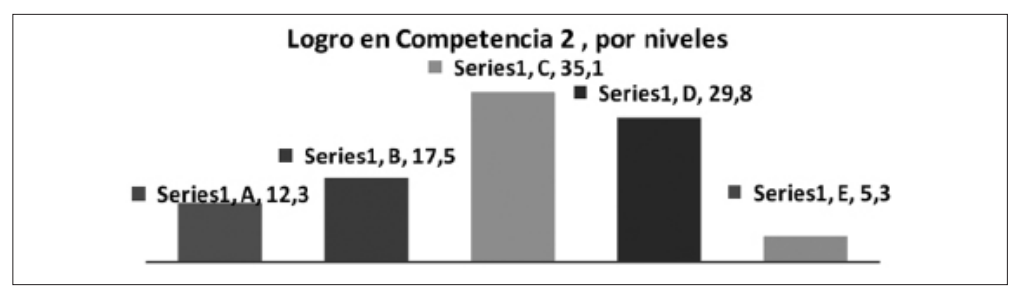


Si se desagregan los resultados según los campos del saber considerados en esta evaluación, vale decir: saber conocer y saber hacer, se distinguirá una cierta diferencia en los logros alcanzados por nivel, entre cada uno de estos campos o dimensiones.

De allí que, como se muestra en el Gráfico 3, en la dimensión del "saber conocer", un 71,9\% (41 casos) alcanza un resultado igual o superior al mínimo aceptable (niveles C, D y E); manteniéndose la concentración mayor en el nivel C, con un 42,1\% (24 casos). En los niveles superiores, nivel D y E, se ubica un 29,8\% (17 casos). $\mathrm{Y}$ en los niveles inferiores (nivel A y B) un 28,1\% (16 casos). A distinción de los logros por niveles alcanzados en la Competencia 1 y Competencia 2, el porcentaje registrado en el nivel más bajo, nivel A, es de sólo un 1,8\% ( 1 caso); apreciándose un logro mayor, respecto de estas competencias, en el nivel superior (nivel E), de un $15,8 \%$ ( 9 casos).

\section{Resultado dimensión del saber conocer, por niveles de logro}

Gráfico 3

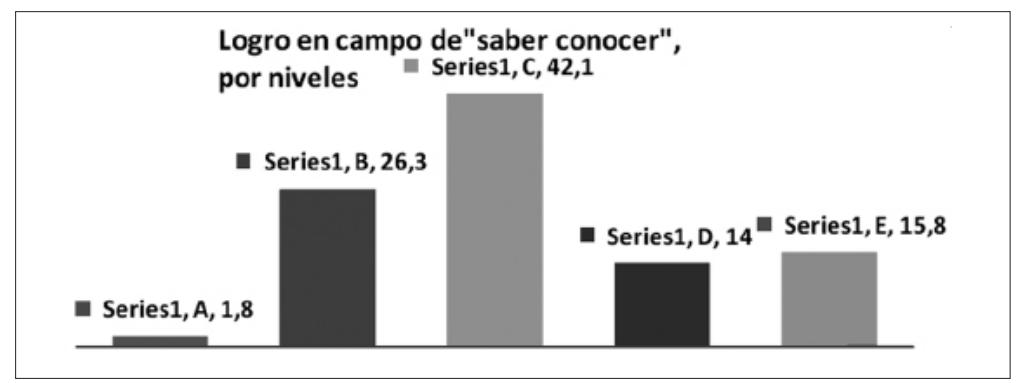

Por su parte, en la dimensión o campo del "saber hacer", un 71,9\% (41 casos) tiene logros iguales y superiores al mínimo aceptable, niveles C, D y E. En esta dimensión la concentración en el nivel $\mathrm{D}$ es mayor que en la dimensión anterior y que en los logros de la Competencia 1, correspondiendo a un 29,8\% (17 casos). De allí que en los dos niveles superiores, nivel D y E, se encuentra un $36,8 \%$ (21 casos), siendo éste el porcentaje más alto alcanzado en estos dos niveles en la evaluación desarrollada. En los niveles inferiores, nivel A y B, se encuentra un 28,1\% (16 casos). Al igual que en la dimensión del saber conocer, en ésta sólo un 1,8\% ( 1 caso) se ubica en el nivel más bajo, nivel A. 
Resultado dimensión del saber hacer, por niveles de logro

Gráfico 4

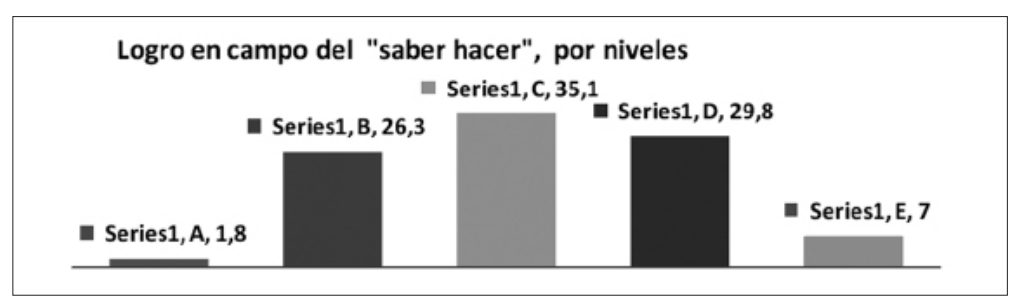

\section{Conclusiones}

Siendo ésta una experiencia piloto de evaluación, el proceso desarrollado adquiere tal relevancia como fuente de aprendizaje para los actores involucrados, que cabe plantear algunas reflexiones al respecto:

- El estudio evaluativo ha permitido determinar que en la población estudiantil sometida al estudio, el logro de las competencias consideradas se ubica dentro de niveles satisfactorios (nivel C, D y E). Vale decir, se constata que la mayoría de los casos evidencia un desempeño equivalente, superior y sobresaliente respecto del mínimo aceptable por la prueba aplicada. Esto permite postular que el Plan de Formación vigente ha favorecido el desarrollo de dichas competencias esperables en este primer ciclo.

- La evaluación de perfil intermedio constituye una oportunidad para re-construir un proyecto pedagógico común. El proceso evaluativo va enfrentando la necesidad de re-discutir aspectos del Plan de Formación: indicadores de distinción entre perfiles intermedios y entre éstos y perfil de egreso; nudos o claves en los recursos pedagógicos; medidas de mejora coadyuvantes para el logro de los perfiles, entre otros.

- A su vez, evidencia la necesidad de reflexión y diálogo en torno a las trayectorias de aprendizaje y rutas de evaluación en la formación de trabajadores sociales.

- Permitey exige re-pensar lagestión de la calidad del aprendizaje. Vale decir plantearse cómo y de qué modo estamos o no favoreciendo los aprendizajes de nuestros estudiantes, cuáles serían los posibles factores potenciadores, con qué otros recursos pedagógicos podemos entrelazar nuestra propuesta de formación, qué coordinaciones y sinergias producimos y 
cuáles son las necesarias, cuáles son los trasvasijes necesarios entre los recursos pedagógicos que ofrecemos.

- Las cuestiones anteriores evidencian la necesidad de articular la evaluación como una acción permanente de la gestión académica. Requiere constituirse en parte de ella, y proveer entonces los mecanismos, recursos y tiempos necesarios para ello.

- La evaluación, como acción cotidiana y reconocida por los docentes, puede contribuir a la generación de sentido de comunidad pedagógica en las Unidades Académicas: dimensionar el comportamiento de las trayectorias de aprendizaje pre-definidas necesariamente coloca la necesidad de plantearse y articular sinérgicamente los diversos recursos pedagógicos que administramos y gestionamos.

- Desde esta perspectiva, la experiencia desarrollada permite visualizar el ejercicio evaluativo en un contexto de aseguramiento de la calidad del proceso de formación profesional.

\section{Referencias bibliográficas}

AlVARADO, V.; CÁRCAMO, A.; GARCÍA, A. (2009). Marco conceptual orientador para el diseño curricular contemporáneo. Diseño Curricular basado en Competencias y Aseguramiento de la Calidad en la Educación Superior. CINDA. Santiago de Chile.

AQUín, N. (2006). ¿Una nueva cuestión social? Revista Perspectivas $\mathrm{N}^{\circ}$ 16. Escuela de Trabajo Social. Universidad Católica Silva Henríquez. Santiago de Chile.

AUDIBERT, M.; CARRASCO R.; DESIMONE P. (2009). Panorama Latinoamericano en torno al Currículo basado en Competencias. Diseño Curricular basado en Competencias y Aseguramiento de la Calidad en la Educación Superior. CINDA. Santiago de Chile.

ESCUELA DE TRABAJO SOCIAL, UNIVERSIDAD CATÓLICA SILVA HENRÍQUEZ (2006). Plan de Formación de la carrera de Trabajo Social. Santiago de Chile. (Documento de circulación interna)

GONZÁLEZ, L.; LÓPEZ, L. (2004). La Sociedad del Conocimiento y la Formación de Profesionales. Competencias de Egresados Universitarios. CINDA. Santiago de Chile. 
GARRETÓN, M. (2000). La sociedad en que vivi(re)mos. Introducción sociológica al cambio de Siglo. LOM. Santiago de Chile.

LECHNER, N. (2002). Las sombras del mañana. La dimensión subjetiva de la política. LOM. Santiago de Chile.

POBLETE, M. Y VILLA, A. (2007). Aprendizaje Basado en Competencias. Una propuesta para la evaluación de competencias genéricas. Mensajero. Bilbao.

TOBÓN, S. (2008). La Formación Basada en Competencias en la Educación Universitaria. El enfoque Complejo. U. Autónoma de Guadalajara. Recuperado en Mayo de 2011. Disponible en: http://www.uag. mx/curso_iglu/competencias.pdf

UCSH (2003). Modelo de formación de la Universidad Católica Silva Henríquez. Documento de circulación interna. Santiago de Chile.

UNESCO (2005). Hacia las sociedades del conocimiento. UNESCO. París.

ZABALZA, M. (2002). La enseñanza universitaria: el escenario y sus protagonistas. Narcea. Madrid.

ZÚÑIGA, M.; POBLETE, A.;VEGA, A. (2009). El Desarrollo de Competencias en un Contexto de Aseguramiento de Calidad. Diseño Curricular basado en Competencias y Aseguramiento de la Calidad en la Educación Superior. CINDA. Santiago de Chile. 